\title{
The D-Scope: Mining the Gap
}

\author{
CAROL MACGILLIVRAY, Goldsmiths, University of London
}

\section{ABSTRACT}

In 2011, through combining practical research into animation and kinetics with digital projection mapping techniques, two students discovered a way of creating apparent motion without using a camera, film stock or a screen. Originally christened the Diasynchronoscope, in acknowledgement of the rich ancestry of pre-cinematic devices of wonder, the $D$-Scope technique involves positioning real objects in a blacked-out acoustic space and illuminating them precisely and sequentially; in effect animating through attention. After some years of refinement, the D-Scope immersive system now carries a registered trademark in the UK. It is an innovative medium, still in its infancy that draws on tropes from animation, film and Gestalt grouping principles to create animation freed from the screen. This paper argues that the technique reveals some new ideas about Apparent Motion when it is experienced and perceived on an environmental scale and not framed by a screen.

\section{KEYWORDS}

Apparent Motion, D-Scope, animation, Gestalt, embodiment

\section{What is Apparent Motion?}

We all know what motion or movement is: A transition of something in space over time. Apparent Motion (AM) is where something does not move, but we commonly perceive it as moving. Most of us experience AM daily as it is responsible for how we perceive film or video running at 24 frames per second (or faster) as a continuously moving picture rather than a sequence of stills. What is less understood, despite being exploited as a phenomenon for nearly 200 years, is why we see Apparent Motion at all.

Some topics cannot help but intersect many fields of study and AM is one of these. This should, by rights, make it a much studied phenomenon; however, in Media Studies (where the text is so frequently inseparable from the vehicle) it is often considered an altogether 'lesser' and more childish medium because of its deep connection to animation. ${ }^{1}$ In Art

\footnotetext{
${ }^{1}$ With the publication of The Illusion of Life: Essays on Animation in 1991, animation finally grew up as an area worthy of study, with editor Alan Cholodenko arguing in his introduction that the profound issues that
} 
History, forms of AM viewed on mechanical devices were regarded as hybrid and lowbrow, secondary to purer art forms such as poetry, sculpture and painting. There are many film theorists who conduct audience-centred research, but very few take a cognitive approach $^{2}$. André Bazin's What Is Cinema? (volumes I and II) have been classics of film studies since publication and are considered the gold standard in the field of film criticism. In his essay on "The Ontology of the Photographic Image" (1971), Bazin makes a case for the definition of cinema being 'Photography that moves'. His argument being that a photograph is a slice of reality, frozen in time. When this idea is extended to cinema, film becomes a medium for conveying reality in time, with filmic images capturing and providing evidence for particular moments in time. This creates a built-in importance on the indexical character of film as a medium of moving images, where the emphasis is firmly on the 'real' image and not the movement.

Although AM has been a fundamental building block for mass media since its inception and is still ubiquitous (particularly more recently in games), there has been a shortage of theorists who straddle media theory and theories of perception. In 1972, in one of the last cross-over discussions of AM as a perceptual phenomenon for film and psychology, electrical engineer Paul Kolers pointed out 'The technology works, but no currently available account of perceptibility of forms in motion is correct. Theory lags practice rather extensively in this domain' (Kolers 1972, x). Since then, there have been few advances made in this area in film studies, and it is fair to say that with a few notable exceptions (Anderson \& Anderson 1993, Bordwell 1997, Steinman, Pizlo, \& Pizlo 2000, Smith 2012), scholars and critics in humanities and the arts have tended to side-step the subject of AM, preferring to leave any questions of movement perception to psychologists and scientists. This paper argues that this is a mistake, because it not only ignores the vital contribution played by the gaps between individual frames - where much is conveyed, but also because it leads to imperfect understanding. Thus film theorists consistently and wrongly attribute AM as a phenomenon to either 'persistence of vision' or flicker fusion. As Joseph and Barbara Anderson painstakingly evidence in two papers, persistence of vision is a myth, which creates an 'inaccurate and inappropriate explanation for the apparent motion found in a motion picture' (Anderson \& Anderson 1993, 1) ${ }^{3}$. Conversely, there has been a reciprocal bias of understanding aspects of universal AM by psychologists, because although film practitioners (particularly animators) have a deep practical understanding of AM, their lack of engagement with theory behind the subject has had an impact on the way that psychologists set about making their own studies. This is particularly notable since the advent of screen-based media where the vast majority of empirical experiments in AM are made using isolated oscillatory movements of flashing

animation raises 'challenge, even suspend, certain axioms of film theory and Film Studies' (1991, 9-10), not least by highlighting the distinction between representation and simulation. Besides, as he points out, 'animation film not only preceded the advent of cinema but engendered it' (Cholodenko 1991, 295).

2 Two exceptions being David Bordwell and Tim Smith who both believe that studies in perception and cognition could create a new paradigm for film theories of spectatorship. (Bordwell 1997, Smith 2012).

${ }^{3}$ They infer that one reason that this misconstruction is maintained is because it lends credibility to the Marxian view held by many film theorists that a film audience is passively receptive $(1993,11)$. 
lights or dots on a screen, rather than taking a more holistic view of AM as a psychophysical phenomenon that draws on proprioception, environment and other sensory inputs.

As pointed out earlier, despite the many studies of AM, theorists have not reached a consensus on why we perceive it. Many psychologists believe AM to be physiologicaly isometric with how we perceive real motion (Robinson 1998, 237), so perhaps we simply perceive AM because we perceive real motion. However Kolers draws a distinction between apparent movement over the retina and real movement in his book concluding: 'For reasons that are still not fully clear, the visual apparatus does not characteristically analyse in detail stimuli that arrive within a brief interval of each other' (Kolers 1972, 185). In other words, all the empirical experiments in AM lead to no solid conclusion one way or the other. This paper argues that in looking for exact measurements of timing and distance on a screen between, say, a flash of light at point $A$, and a subsequent flash of light at point B, psychologists may be looking in the wrong way, because there can be no exact measurement of AM; it is a subjective phenomenon and is consequently impossible to measure without observational context and perceived at scale. The place to look is where the change is made manifest: in the gap between continuous stimuli. ${ }^{4}$

Any research into motion perception requires first creating a device that shows motion in a replicable way. In today's age of the ubiquitous screen that appears an easy task, but the original published research on AM came from observing the phenomenon in the real world.

\section{Gestalt Psychology}

'One sees motion: an object has moved from one location to another. One describes the physical facts: Until time t1, the object was in position pl. From time tn on, it was in position pn. In the interval between 11 and th, the object was successively, continuously in space and time, in the intermediate positions between $\mathrm{pl}$ and $\mathrm{pn}$, through which it arrived at pn. One sees this motion.'

(Wertheimer 1912)

Thus starts the seminal article that gave us the foundations of Gestalt psychology. Max

\footnotetext{
${ }^{4}$ A long time ago, the author edited a film on killer whales bred in captivity where a trainer insisted that Billy, the whale she trained must to be able to read her mind because he seemed to be able to anticipate what she would say before she gave a command. On closer examination it became apparent that Billy (who as a whale communicated using sonar), was interpreting the trainer's breath intake before she gave her orders. The text on which he acted was communicated by the gap preceding the order, an idea that the trainer (who communicated in words) had not countenanced.
} 
Wertheimer's experiments were predominantly made using a 'Tachistoscope,5 and his experiments were concerned mainly with the phenomenon of AM between two stimuli.

In his article, Wertheimer hypothesises that 'a subjective completion' must have somehow occurred between one stationary event and the next. We now know that all perception is subjective: our actual visual perception of the world is derived from small quantities of data, but because that data is supplemented and completed (as Wertheimer hypothesized) subjectively, we have the impression of holding a full view of the real world. In fact, information coming in to the visual cortex is supplemented by much neural feedback traffic travelling back (Eagleman 2015, 33, Schacter 2007). So, rather poetically, our view of the world is shaped according to how we shape it, and how we form ideas of the shape of the world seems to come from a combination of evolution and experience, or how the world has shaped us (Purves et al 2012).

The other founders of Gestalt theory were Wolfgang Kohler and Kurt Koffka. They joined Max Wertheimer in his laboratory at the Psychological Institute in Frankfurt in 1910 to study motion perception. Together they established the school of Gestalt with a view to redefining approaches to psychological research through a set of theoretical and methodological principles. The guiding theories were that of Totality - that conscious experience should be considered globally, by taking into account all the physical and mental aspects of the subject simultaneously - and, more controversially, the theory of psychophysical isomorphism - that there is an exact correlation between conscious experience and cerebral activity.

The Gestalt school founded its methodology of investigation into universal principles for the way we organize perceptual forms by studying responses to actual perceived phenomena in the real world; taking experimentation on subjects out of the lab and attempting to conduct experiments in natural situations and in real conditions to produce a greater fidelity to the real world. They came to the conclusion that structured wholes, rather than isolated sensations, are the primary units of our mental life. In this respect their work corresponds with more recent work in Embodied Cognition and sensorimotor accounts of visual consciousness (O’Regan \& Noë 2001). Gestalt ideas confound mechanistic models of human perception: we psychologically attempt to make order out of chaos, to create harmony or structure from seemingly disconnected bits of information. How we best could receive this information and arrange it is governed by a fundamental of Gestalt theory; the 'law of Prägnanz'. Prägnanz is a German word that has been translated variously as salience or conciseness, but perhaps one of the best conversions from the German would be 'pithiness' (Spillman 2012).

The Gestalt school offers us several Gestalt laws or principles of perceptual organization that illuminate how we perceive and group information in our minds according to

\footnotetext{
${ }^{5}$ A device that displays an image for a specific amount of time, (a bit like a slide projector or a magic lantern).
} 
Prägnanz; e.g. Figure/Ground articulation, Good continuation, Closure etc.

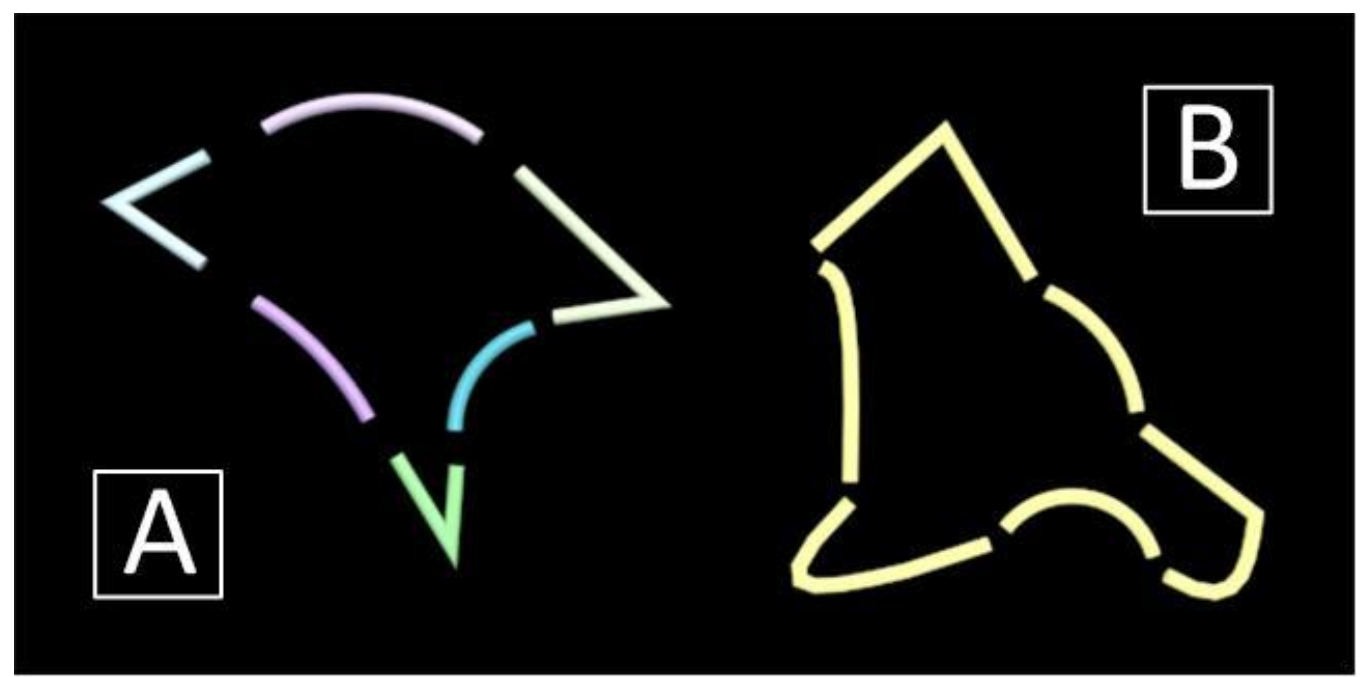

Illustration of the good continuation principle. Figure A, has good continuation (although it is an unusual shape and the elements are different colors and the gaps between larger) because the edges of the lines are aligned and the figure lines flow easily across the gaps to each other. In figure, B, the flow of the figure is disrupted, making it difficult for a viewer to reproduce from memory. (@) Author)

Most of these laws deal with how we reify gaps into wholes and they are not limited to static visuals, but include motion, auditory and tactile Gestalt. Many of these structural patterns, when combined with the subjective nature of phenomenal experience, are fundamental to how we perceive AM, and they offer insights into how to design patterns for optimum communication and construct sequences of stimuli so that they appear to move. Gestalt psychology was founded on global research, a desire for 'wholeness', but today academia tends to segregate subjects into discrete disciplines, and these indexical boundaries mean we have lost much of the cross-fertilization between science and art, practice and theory and invention and open-sourcing that inspired so many pioneers in the illusion of movement. ${ }^{6}$

\section{Two sorts of Apparent Motion; Phi and Beta}

The ground-breaking motion study credited with founding Gestalt psychology was Max Wertheimer's discovery of phi movement in 1912 (Wertheimer 1912). The breakthrough came from an experiment he constructed after observing the appearance of movement

\footnotetext{
${ }^{6}$ Hybridity carries repercussions: Goethe's experiments with colour in the early 19th century created quite a stir with artists and in the next century many colour organs were made which attempted to make coloured visualizations of music. The second Color/Sound Congress in 1930 in Hamburg was attended by psychologists, scientists and cultural studies scholars as well as artists, pointing to its true hybrid nature and for a whole decade, several color organs were demonstrated at this series of Color Music Congresses. All these devices have perished together with their inventors and are forgotten, lost in their own complexity and interdisciplinarity (Daniels, 2011, 11).
} 
generated by alternating railway signals on a train journey he took to Frankfurt. Although derived from real-world experience, Wertheimer's experiments were predominantly made using a tachistoscope, and his experiments were concerned mainly with the phenomenon of AM between two stimuli. However, he did conduct some experiments where three or more elements were used, where he concluded that the movement between two elements is strongly influenced by the presence of neighbouring elements.

Wertheimer published on the phi phenomenon, branding it 'pure' motion, because although the perception of movement is clear, the subject of the motion is absent. This was a groundbreaking study because it was the first to suggest that we could observe motion without shape or a central object of attention, and that means that motion is perceived in its own separate pathway. ${ }^{7}$

Phi movement is a difficult concept, and understanding of the phenomenon was not helped by misprints in the translation of Wertheimer's study at the time (Steinman, Pizlo, \& Pizlo 2000). This has led to some media theorists attributing AM in movies to phi movement combined with persistence of vision, when in fact screen-based moving images are, by and large, constructed of beta movement, and persistence of vision is a proven misconstruction (Steinman, Pizlo, \& Pizlo 2000) (Anderson \& Anderson 1993).

This study will return to phi movement and discuss its importance in context with the DScope, in a case-study artwork that explores the difference between phi and beta movement, Gestalt Circle, created as a centennial piece in 2012.

\section{The heritage of the D-Scope}

'The D-Scope ${ }^{\circledR}$ is 'an audio-visual apparatus which creates a system of apparent motion using concrete physical objects lit sequentially'

(Trade Marks Registry 2014)

Fundamental to the D-Scope technique is the idea of using projection to illuminate threedimensional objects, thereby preserving features of perspective, focus, depth perception and parallax in an apparatus that can be both immersive and interactive (MacGillivray \& Mathez 2013). Although the artworks are digital in construction, relying on software and computer visualizations, the sculptural elements and their positions are in effect 'backwards engineered' and concretely constructed in space and so are firmly analogue. For audiences, it seems to evoke sensations similar to descriptions of encounters with devices of wonder from days of pre-cinema and even further back (MacGillivray \& Mathez 2012). Because the D-Scope is so hybrid, comprehensive documentation of all of

\footnotetext{
${ }^{7}$ Some credit for Wertheimer's discoveries must go to Viennese physiologist, Sigmund Exner, with whom Wertheimer had conducted his post-doctoral research. It was Exner who first propounded the idea that we could see motion out of the corner of the eye with no shape or colour (Exner 1875).
} 
the preceding devices in pre-cinematic age would be impossible in this essay: the devices of wonder in the nineteenth century found inventors, entrepreneurs, artists and scientists leap-frogging each other with ideas to put before an audience that was ready and willing to be both observer and observed, consumer and participant, becoming part of a system that blends science, art and spectacle (Crary 1992, 119-130). The two most popular and influential devices were the zoetrope (and its successors) for viewing animations and the magic lantern for projecting images.

The first known machine which worked as a unit to create simulated movement was an early 'zoetrope' invented in China by Ding Huan in the late Han dynasty (202BC - 220 AD). The name 'Zoetrope' comes from the Greek, 'Zö̈' (life) and 'Tropos' (turn) and is usually translated as the 'Wheel of life'. The zoetrope is often cited as a precursor to cinema, because it is the first recorded manifestation of AM. However, it is a fairly limited device for viewing evenly spaced, looped animations only. Ding Huan's invention used convection from a lamp to turn vanes attached to a round drum of translucent paper panels on which sequential pictures of flying birds and other animals had been painted; the lamp illuminated the pictures as the drum rotated, creating an illusion of movement, much as children's rotating night-lights do today.

The zoetrope has a rich history in Europe, going through several incarnations in the 19th century. It was a popular parlour toy from 1833 when William George Horner customised it as the 'Daedalum' or 'Wheel of the devil', and strips of pictures (usually 15) that fitted in the drum were sold commercially. Horner's Daedalum displaced the phenakistoscope ${ }^{8}$, a construct made in 1829 by Belgian physicist Joseph Plateau. Plateau constructed the device to conduct research on Newton and Rogets' ideas on persistence of vision ${ }^{9}$ using viewing slits to segment the images. Because the slits provide an effective 'shutter' effect, the phenakistoscope is also cited as the godfather of film, and it is Plateau who can be credited with the birth of the myth of persistence of vision. The phenakistoscope used flat discs of 10-16 pictures. The resulting movement could only be seen by one viewer at a time and needed a viewing mirror, which is why the zoetrope proved more popular - although this in turn was improved upon in 1877, when Charles-Emile Reynaud introduced the 'praxinoscope', which allowed for longer shows and produced a brighter, less distorted image.

Eadweard Muybridge's 'Zoopraxiscope' (1880) built on Reynaud's device, and it is welldocumented how he used a series of trip wires set at regular spaces on a series of cameras to record a horse running (Clegg 2007). The zoopraxiscope projected images from a rotating glass disk to give the impression of motion. The stop-motion images were initially painted onto the glass as silhouettes. A second series of discs, made in 1892-1894, used outline drawings printed onto the discs photographically, then colored by hand. The

\footnotetext{
${ }^{8}$ The name phenakistoscope was also derived from Greek origins and can be translated as 'eye deceiver'.

${ }^{9}$ Roget had presented paper on the subject to Royal Society based on the optical deception created by viewing a revolving train wheel seen through slits of a fence (Roget 1825).
} 
resulting drawings are held up even today as realistic animations, although it is worth pointing out that the spacing of the pictures was due to distance and not time, so if used to record a slower or more uneven motion, it would perhaps be noticeable that the timing of the animations is as not 'realistic' as they are generally regarded to be, because realistic animation involves movement in time and space, and both variables are needed for accurate interpretation of movement.

As Muybridge had studied horses and human motion, Jules Etienne Marey was keen to uncover the secrets of biological motion in birds. His innovative, photographic gun recorded birds in flight (1882) and is in many ways the closest precursor to the D-Scope, not least because he also worked in a blackout space. Marey hung black velvet drapes inside a shed at the Physiological Station in Paris, and used a photographic 'rifle' to follow white birds in flight. The images were recorded on discs with radial slots to act as shutters between pictures. This use of white against a black velvet background, and of progressive animation that is attended to (by the rifle sighting), makes his animations a close 2D cousin to the animation by attention that is achieved in the $D$-Scope.

The invention of the 'magic lantern' as a way of projecting images painted on glass slides onto a wall is generally credited to Dutch astronomer and mathematician, Christiaan Huygens in 1656, although there are pointers to it being an earlier invention. It used a concave mirror behind a light source to direct as much of the light as possible through a small rectangular sheet of glass on which was painted an image. The image on the magic lantern slide was thus projected through a lens at the front. The lens was adjusted to focus on a projection screen or wall. Initially, candles or oil lamps were used, producing very dim projections. Improvements in lighting took the form of the Argand lamp from the 1790 s, limelight in the 1820 s and the arc light in the 1860s. The audience came to enjoy the magical properties of the lantern in two ways; as instruments of illusion and also as replicators of reality. The slides could be used to promote the wonders and beauties of the world, or summon up phantasmagorical multi-media experiences. This bifurcation of education and entertainment was evident from early on, and there were warnings to exhibitors that they should take great care to inform spectators that such images were naturalistic and not magical in origin.

The pre-cinema age was a gift to entrepreneurs who were quick to see the commercial advantage of combining popular parlour devices of animation with the magic lantern and by 1870 the major manufacturers, Peck and Snyder, boasted at least 47 different varieties of Magic Lantern. The most popular was the Electro Radiant no 2, which was sold with bundled software of 12 × 7" slides. They were lit (hazardously) with oil, gas, or kerosene and came complete with a smoke stack! The projected images from slides were $8 \mathrm{ft}$ in diameter, allowing for a sizable audience and a major selling point was to encourage entrepreneurial boys to get one and sell tickets. Apparently skilled projectionists could 
create a semblance of movement by moving swiftly from one slide to another. ${ }^{10}$

From the mid- to late-1800s, Victorian parlours must have been fit to bursting with mechanical toys. Alongside the magic lantern, there was the 'Zograscope' from the 1740s which was a means of curving a scene in a bowl-like way away from the viewer, so suppressing flatness in favor of depth cues. When the zograscope was held at arm's length, the scene would 'pop' into a life-like facsimile. Another device was the 'Lithophane' of 1822 , where an image was carved in low relief into a porcelain plate and backlit to produce an image of 'great depth and realism'. In 1849, David Brewster had invented the lenticular 'Stereoscopic' that resembled opera glasses for viewing paper prints or stereographs. Queen Victoria very much admired Brewster's stereoscope as a tasteful and didactic tool at the 1851 Great Exhibition, and such was its ensuing popularity that within six years the London Stereoscope Company has produced 100,000 stereographs; these devices were as ubiquitous as televisions are today. Alongside these, from 1886, every wealthy home in Britain had a 'Kinora'. This was a device for making short home movies (up to 25 secs long) and it worked like a flip-book on a wheel. The English company, Kinora Ltd., offered a camera that shot rolls of $25.4 \mathrm{~mm}$ paper or celluloid which were then processed and made into reels by the company. They also sold ready-made movies for people to buy or rent. ${ }^{11}$

The mechanical devices of this pre-cinema age were gradually replaced by screen-based content, and the screen has reigned supreme for most of the last century. However the exploratory heritage of mechanical animation devices where each new medium was as thrilling as the content it held, has been subject to modern revisitations and revisions as it seems that they continue to hold a fascination for us. The D-Scope (originally christened the 'Diasynchronoscope' in a nod to its mechanical ancestors) is one such medium.

\section{What does the D-Scope do?}

Used in a performance environment, the D-Scope is a new tool for investigating the expressive power of embodied screen-less animation. It takes place in an architectural black-out where prepared 3D objects are arranged in a way that they change incrementally in shape and/or position. Although nothing in the space actually moves; the objects are revealed through serial illumination in the dark room, sequenced using the technique of projection mapping. This technique enables creation of a dramatisation over time,

\footnotetext{
${ }^{10}$ The same ability was reported in accounts of the tachistoscope experiments that Wertheimer (1912) and Metzger (1934) made when comparing real and apparent motion.

${ }^{11}$ Even with the invention of cinema by the Lumière brothers, in 1895, the Kinora remained a hugely popular gadget until 1914, as for a long time it was deemed socially unacceptable for genteel folk to go to the cinema. The animation artist George Griffin still employs the techniques of the Kinora in his some of his interactive artworks and a modern version of these mechanical flipbooks made as triptychs, were exhibited by Californian Artists, Mark Arnon Rosen and Wendy Marvel at Kinetica Art Fair 2013. In a talk at the fair, the pair described how unexpectedly popular they were with modern audiences as gifts. Their work can be seen at http://xraydreams.com/
} 
encouraging the common perceptual shortcuts of a participant to create sequences and a narrative from static abstract objects. Because it is an immersive medium, it is far harder to describe on paper than to see. It differs from film because there is no screen or camera, it differs from an immersive game (such as Oculus Rift) as it serves an audience of more than one and there is no intervening head-set or glasses or compression of what is seen, and it differs from mechanical devices because, being sculptural, there is no image. It differs from a hologram because it takes place over time and there is AM.

In the D-Scope, the gaps between objects are two-fold: first, in distance, where objects positioned further apart apparently move faster; and secondly, in duration of attention, where objects are illuminated for discreet times. The gaps between each illuminated object form the narrative and structural essence of the experience.

The closest cousin for the $D$-Scope is a ' $3 D$ zoetrope'. The first known original $3 D$ zoetrope was made by Jules Etienne Marey, and consisted of small, sculpted birds flying within the zoetrope drum; the birds were viewed through a slit in the drum and so the sculptural element was not the most important part of the machine and, indeed, it is seldom referenced today. The American artist Gregory Barsamian turned Marey's 3D zoetrope 'inside out'. He creates large-scale turntables, which, when lit by strobe lighting synchronised with the speed of rotation, generates the illusion of 3D models animating in a real space. Barsamian exhibited a large-scale $3 D$ zoetrope, Die Falle (The Trap) at Kinetica Art Fair in 2013. The review by Mathew Tucker in the Huffington Post noted:

\begin{abstract}
'Barsamian's exhibit [...] uses strobe lighting to create jerky mesmerising movement... Taking on the visual illusion known as 'persistence of vision', the sculptures are perceived in real time but appear within a dream worl.' (Tucker 2013).
\end{abstract}

The movement is 'jerky' in a $3 D$ zoetrope because of the strobing required to create the illusion and there is no narrative or synchronized sound, just noisy, mechanical looped action, but still $3 D$ zoetropes are objects that create great wonder in modern spectators. They feel both mesmeric and magical. Pixar and Studio Ghibli have each created versions featuring 3D figures from their animated films to wonderful effect.

The D-Scope medium offers a unique new experience to audiences: the light is constant and the animation is smooth, so the impression is of real objects magically moving and interacting in space. Although it often uses loops of animation, it can be used to construct dramatic progression. The sound is situated and synchronous, and audiences can move freely around in the shared space, making the experience immersive and of a human scale. In the D-Scope it is possible to create variables in time and space, and to animate in phi or beta AM. The D-Scope uses existing techniques, but they are combined in such a way that they supply a new context for addressing questions in perception and the aesthetics of AM (MacGillivray 2014). 
In shaping our scientific and artistic exploration, we revisited Gestalt and animation principles, and looked at ways of integrating early experimental techniques for investigating AM that used a consistent light source with modern digital software techniques. An early artwork, Gestalt Circle, was constructed as an architectural extension of Gestalt founder, Max Wertheimer's experiments in phi and beta movement, deconstructing the two versions of AM by integrating them.

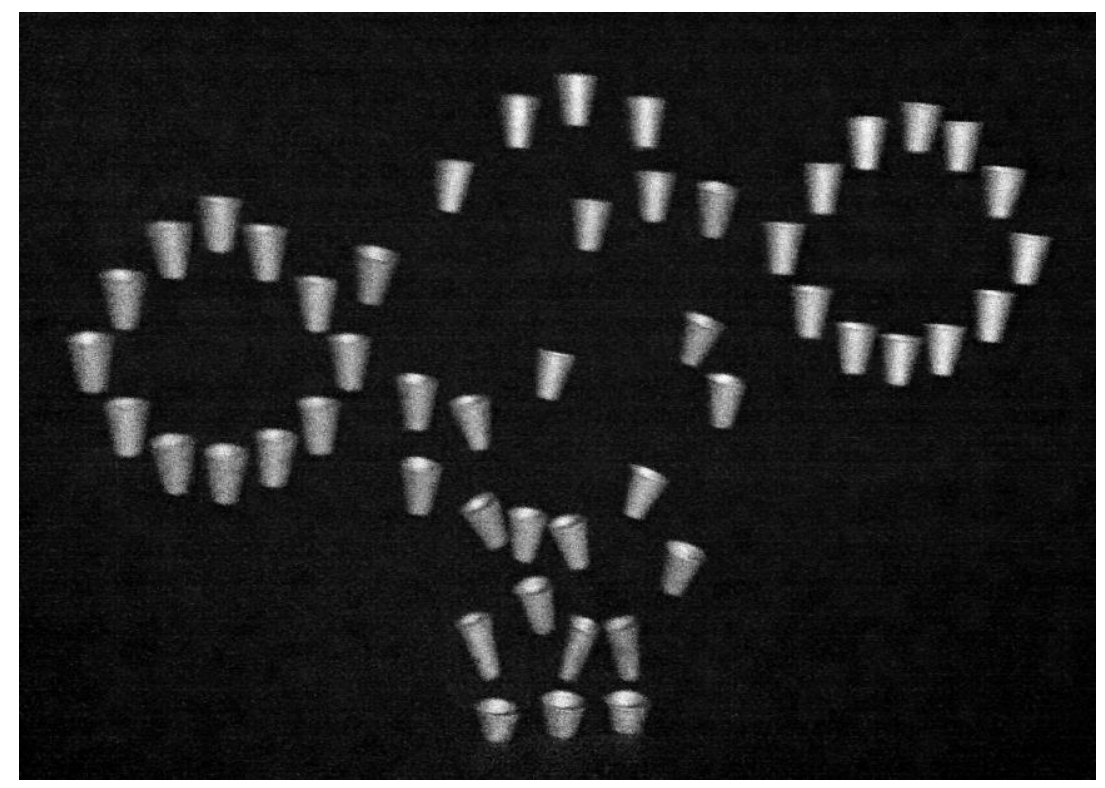

Gestalt Circle - The holistic view of time choreographed in space - not seen by the audience (@) Author)

\section{Case Study: Gestalt Circle}

'Gestalt Circle synthesizes digital and analogue technology: the nonrecyclable coffee cups are resuscitated and reframed by digital technology, allowing the artists to re-animate throwaway objects in a unique, ghostly ballet, using the Diasynchronic technique.'

(Kinetica Art Fair catalogue 2012)

Gestalt Circle, a hybrid of experiment and art, was shown to about 1500 visitors at the Kinetica Art Fair 2012 in London, UK. Film of the artwork is available at http://www.trope-design.com/exhibited-artworks/gestalt-circle/. Wertheimer had dubbed phi as 'pure' movement because the perceiver's attention does not centre on a concrete object, but rather on the absence of an object. In this centennial meditation, multiple polystyrene coffee cups were arranged in space, then lit in sequence to create apparent movement in two ways: beta and phi. The individual (beta) movement - free, organic and playful - breaks away from the regimented and replicated (phi) mechanical movement and then returns to it in a continuous cycle. The artwork had a hypnotic quality, and was so popular by the end that we had to limit times for audiences to 3 minute's duration. 
Gestalt Circle was the first exhibited artwork made using the D-Scope. During the following four years, there have been twelve exhibited artworks, alongside many experiments which progressively interrogate the D-Scope as new medium. For instance no one has seen phi motion with real objects acting as stimuli before.

The biggest contribution that the D-Scope makes to studies of AM is to add an environmental context to it, bringing with it global notions of combined sensory input. There is no doubt that humans are particularly sensitive to biological motion, but it has also been suggested that this sensitivity is comparable to the sensitivity to structured nonbiological motion (Hiris 2007, Nishida 2011). One of the medium's unique attributes is combining environmental animations with precisely synchronized sound. Sound is treated as a formal raw material that is morphologically equivalent to the iconic objects placed and lit in the D-Scope, intertwining sound and visuals in a gestalt beyond that of most screenbased media. The synthesis of sound and vision is a primary element of much of any animation's appeal, and was investigated at length by Michel Chion who coined the term for it of 'Synchresis', defining it as: 'The forging of an immediate and necessary relationship between something one sees and something one hears' (Chion 1994, 5). Undoubtedly synchronized and spatialised sound alongside embodied proprioception play a large part in increasing saliency in perception of movement.

Motion perception of a scene depends not only on retinotopically extracted motion signals but also on how those motion signals are assigned and interpreted. In fact it has often surprised us in later artworks how little stimulus we need to provide to communicate continuous motion. One conjecture is that participants may not be watching the lit objects in focus, but parafoveally. This means that their eyes are leading the action, which is a common occurrence in smooth pursuit. It also can be found in the way we read words on a page, where we read 14-15 letters ahead (to the right if reading English, to the left if reading Hebrew) using parafoveal information. This allows us to 'preview' what lies ahead to see if it is a word or not. The duration of this access is 50-150 milliseconds (Wolf 2007). We start to explore our semantic comprehension of a word later, from 200-500 milliseconds $(2007,157)$. Extrapolating back from this to the $D$-Scope, where one frame is equal to $41.6 \mathrm{~ms}$ (or $83.2 \mathrm{~ms}$ if running at 2 frames per second), it is perfectly possible for participants to be picking out up to three objects at once when moving at $24 \mathrm{fps}-$ even though the objects are being lit sequentially. This is consistent with what we have found in interviews with spectators when perceiving a bouncing block, where they reported that they had the sensation of seeing multiple blocks at a time. It also may explain the persistent misattribution of AM to 'persistence of vision' - what we are really perceiving is a persistence of mind and memory not 'ocular lag' on the retina. ${ }^{12}$

\footnotetext{
12 This fits with the retrospective construction theory of seeing movement put forward by some psychologists (Kuhn \& Rensink 2016, van der Waals \& Roelofs 1931), or the combined predictive and retroactive explanation (Maus \& Nijhawan 2006), where they suggest that the brain combines predictive mechanisms with employment of retroactive mechanisms to come up with the most reasonable interpretation of the sensory input when confronted with unpredictable events.
} 
This may also, in part, explain why the D-Scope is frequently described as 'hypnotic' - it could be an effect produced by participants not actually focusing on anything at all, but having a visual experience that is entirely parafoveal. If this were the case, it would be interesting because we would be achieving a new form of parafoveal attention using installations where nothing moves. When perceiving movement the unattended gaze usually picks up on a new onsetting stimulus parafoveally, and then tracks it. This is considered a physiological (bottom-up) phenomenon. In the D-Scope, the perceived stimuli that are perceived as AM would be 'behind' a participant's gaze which is travelling ahead along a predicted path. Because such perception would be psychological and 'top-down' this supports the ideas of several visual psychologists (Lu 1995b, Marr 1982, Petersik 1991, Kinchla \& Wolfe 1979) and art theorists (Boden 2010, Crary 1992) and suggests that, rather than being passive receivers, viewers are not merely spectators, but participants carrying an interpretative, 'exegete' status when viewing objects in AM within the $D$ Scope; discovering the text of the movement by interpreting the gaps between the objects as intervals of distance and time.

Artistically this raises another interesting question to be considered: what is material in an artwork? This is particularly of interest in the case of Gestalt Circle, where participants 'seeing' phi movement are following a non-object and perceiving movement of a 'noncup'. This means that is not just material sculptural objects that are used for artistic purposes, but also the 'immaterial' is fundamental to the artworks. Most obviously Gestalt Circle is made from polystyrene cups, but it is also made from:

- Polystyrene cups (which are painted black, but perceived as white) - plus

- Sequenced projected light

- Speakers and audio

- Foam, wood, metal, cloth (to make the underlying structure)

- Computer hardware and software

In addition it could be argued that further relevant 'immaterials' are that the multiple polystyrene cups are:

- Arranged 'diasynchronically'(i.e. using the D-Scope technique)

- In a blackout space, and

- Require participant observation

These 'immaterials' are fundamental because, in the D-Scope, the gap is not absence - it is its pure structural essence - and it is through embodied perception of these gaps that participants perceive/create AM. In Gestalt Circle, during the depicted phi movement, participants are following the immaterial gap and not a material cup, thus we have an example of 'Meta-Merz ${ }^{\text {,13 }}$ where the material (conceivable anything) is combined with the

\footnotetext{
${ }^{13}$ After Dadaist Kurt Schwitters' paradigm of 'Merz'; bringing every conceivable material together for artistic purposes, with each individual material (both aural and concrete) contributing to the same effect in equal part.
} 
immaterial (conceivable nothing) and the inconceivable (what you see is not what you get).

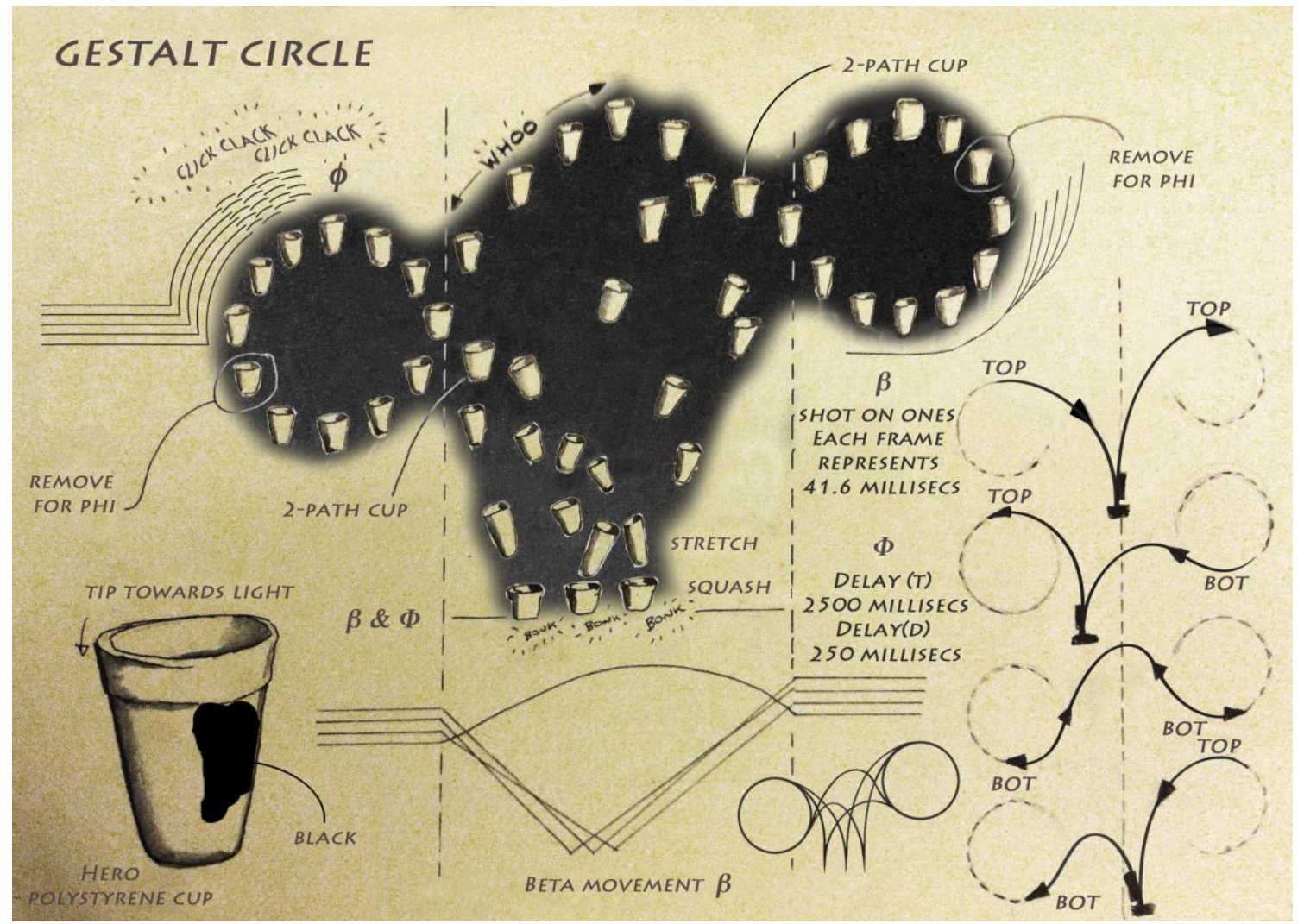

Schematic of Gestalt Circle (C) Author)

\section{Summary}

Each art has a medium and that medium is especially fitted for one kind of communication. Each medium says something that cannot be uttered as well or completely in any other tongue.

(Dewey 1934, 11)

If the story of how artists, scientists and inventors have sought to create and capture AM through the ages teaches us anything, it shows that all definitions of AM can be re-thought in the context of changing technology. The D-Scope is an immersive installation that draws upon plastic arts, Gestalt theory and animation techniques to do away with the screen and evoke a bygone age of optical fascination for an audience as they experience a new embodied and synaesthetic medium. This is a new way of seeing AM in a new medium and there is still much more to explore. So far, D-Scope artworks have been shown to over 10,000 people and gained appreciative testimony from participants of all ages, experts, reviewers in art and science and also from other artists (MacGillivray 2014). Although the research is practice-based, and as such the artefacts play a significant role in that research, 
the new knowledge that this study pursues is not found in the artifacts in and of themselves, but in chronicling the formalisms for this new medium as they are discovered, and then contextualizing these formalisms with other practice. It is hoped that the hybridity of the $D$-Scope will not consign it to becoming the same unread footnote of history as the multiple colour/sound organs of Hamburg.

This research contends that the spatiotemporal nature of the $D$-Scope offers a unique framework for creating principles of non-biological apparent motion in an ecological environment, in other words that the medium comes with its own grammar, in the shape of formalisms which have been developed through studio practice and tested in exhibited artworks, some of them are new and some of them, as has been detailed here, can find their roots in formalisms of early film and the three focal theories of Animation, Gestalt theory and Perceptual Psychology.

It is the contention of this essay that as a new medium, the D-Scope offers some interesting new perspectives on perception of AM that consequently have impact on theories of spectatorship in film theory and arts practice, and could potentially lead to review of empirical experiments in AM in science, where movement is treated in a more environmental way. In particular it urges both groups to look beyond the indexical, and view the D-Scope creations as manifestations of the importance of the gaps between things; because it is in these spatiotemporal gaps that the secret of the perception of AM lies.

\section{References}

Anderson, J., \& Anderson, A. (1993) 'The Myth of Persistence of Vision Revisited', Journal of Film and Video, 45(1), 3-12

Bazin, André. (1971) What is cinema? Vol. 1 \& 2 (Hugh Gray, Trans., Ed.), Berkeley: University of California Press

Boden, M. (2010) Creativity and Art: Three Roads to Surprise, Oxford: Oxford University Press

Bordwell, D. (1997) Post-Theory: Reconstructing Film Studies, Madison: University of Wisconsin Press.

Chion, M. (1994) Audio-Vision:Sound on Screen, New York: Columbia University Press.

Cholodenko, A. (1991) 'Who Framed Roger Rabbit, or the Framing of Animation' in A. Cholodenko (ed), The Illusion of Life. Essays on Animation. Sydney: Power Publications

Clegg, B. (2007) The Man Who Stopped Time: The Illuminating Story of Eadweard Muybridge, Washington DC: Joseph Henry Press

Crary, J. (1992) Techniques of the Observer: On Vision and Modernity in the Nineteenth Century, London: MIT Press 
Daniels, D. (2011) 'Prologue: Hybrids of Art, Science, Technology, Perception, entertainment, and Commerce at the interface of Sound and Vision' in D. Daniels (ed), See This Sound: Audiovisuology 2 - Essays, New York: Verlag der Buchhandlung Walter König pp. 9-25

Dewey, J. (1934 (reprint 1989)) 'Art as Experience' in J. Boydston (ed), John Dewey: The Later Works, 1925-1953, Carbondale: Southern Illinois University Press, p.10

Eagleman, D. (2015) 'The Brain: The story of you', Edinburgh: Canongate Books

Exner, S. (1875) 'Experimentelle Untersuchung der einfachsten processe - III', (Experimental research on simple physical processes - III). Perflugers arciv fur die Gessampte Physiologie des Menschen und Thiere, 11, pp. 403-432.

Hiris, E. (2007) Detection of biological and nonbiological motion. Journal of Vision, 7(12): 4, pp.1-16

Trade Marks Registry, Intellectual Property Office, (2014) Certification to Trope LLP of D-Scope®. Registered in Great Britain and Northern Ireland. Ref No: UK 00003047681 available at https://www.ipo.gov.uk/tmcase/Results/1/UK00003047681?! accessed 17 May 2016

Kinchla, R. A. \& Wolfe, J. M. (1979) 'The order of visual processing: "Top-down," "bottom-up," or "middle-out"', Perception \& Psychophysics Vol. 25 (3), pp. 225231

Kolers, P.A. (1972) 'Aspects of Motion Perception', in H.J. Eysenck (ed) Vol.16 of International Series of Monographs in Experimental Psychology, Oxford: Pergamon Press Ltd.

Kuhn, G. \& Rensink, R.A. (2016) 'The Vanishing Ball Illusion: A new perspective on the perception of dynamic events' in Cognition, available at Epub 2016 March, accessed 10 October 2016, pp. 64-70

Lu, Z-L. \& Sperling, G. (1995b) 'The functional architecture of human visual motion perception' in Vision research, 35, pp. 2697-2722

MacGillivray, C. (2014), Thesis. Goldsmiths College (University of London) available at http://research.gold.ac.uk/10850/ accessed 16 May 2016

MacGillivray, C. \& Mathez, B. (2012) 'Developing a system of screen-less animation for experiments in perception of movement', Leonardo Electronic AlmanacLEA Volume 18 No. 3, Touch and Go Volume.

MacGillivray, C. \& Mathez, B. (2013) 'The Diasynchronoscope: Bringing a new dimension to animation', Confia (International Conference on Illustration and Animation), Porto: Edicao IPCA pp. 367-379

Marr, D. (1982) Vision: a computational investigation into the human representation and processing. San Francisco: W.H. Freeman

Maus, G.W. \& Nijhawan, R. (2006) 'Forward displacements of fading objects in motion: The role of transient signals in perceiving position' in Vision Research (Dec), Elsevier

Nishida, S. (2011) 'Advancement of motion psychophysics: Review 2001-2010', Journal of Vision 11(5):11, available at 
http://jov.arvojournals.org/article.aspx\%3Farticleid=2191813 accessed 16 May 2016, pp.1-53,

O'Regan, J. K. \& Noë A. (2001) 'A sensorimotor account of vision and visual consciousness', Behav. Brain Res.24, pp. 939-1011

Petersik, J. T. (1991) 'The two-process distinction in apparent motion', Psychology Bulletin 106, pp.107-127

Purves, D., Cabeza, R., Huettel, S.A., LaBar, K.S., Platt, M.L. \& Woldorff, M. (2012) Principles of Cognitive Neuroscience (2nd edition), Sunderland, MA: Sinauer Associates

Robinson, J.O. (1998) The Psychology of Visual Illusion (Dover edition, original 1972), Mineola, NY: Dover Publications, Inc.

Roget, P. M. (1825). 'Explanation of an optical deception in the appearance of the spokes of a wheel when seen through vertical apertures', Philosophical Transactions of the Royal Society of London, Vol 115, pp.131-140.

Schacter, D.L. Addis, D.R. \& Buckner, R.L. (2007) 'Remembering the past to imagine the future: the prospective brain', Nature reviews Neuroscience 8(9) pp. 657-61

Smith, T. J. (2012). 'Exploring Inner Worlds: where cognitive psychology may take us' in I. Christie (ed), Audiences, Amsterdam University Press.

Steinman, R., Pizlo, Z. \& Pizlo, F. J. (2000) 'Phi is not beta, and why Wertheimer's discovery launched the Gestalt revolution', Vision Research 40, pp. 2257-2264.

Spillman, L. (2012) (Ed. Trans.) Wertheimer, On Perceived Motion and Figural Organisation (From M. Wertheimer, 1912, article in Zeitschrift für Psycologie).

Tucker, M. (2013) available at http://www.huffingtonpost.co.uk/2013/03/01/kinetica2013-artfair_n_2789402.html accessed 8 Jan 2016, Huffington Post.

van der Waals, H.G. \& Roelofs, C.O. (1931) Optische Scheinbewegung Zeitschrift für Psychologie und Physiologie der Sinnesorgane 115, pp. 91-190

Wertheimer, M. (1912). Experimentelle Studien uber das Sehen von Bewegung. Zeitschrift fur Psychologie, 61, 161-265.

Wolf, M. (2007) Proust and the Squid: The Story and Science of the Reading Brain, New York: Harper Collins 